\title{
O PAPEL DAS ESPÉCIES REATIVAS DE OXIGÊNIO NA FOTÓLISE DA ATRAZINA
}

\author{
M. P. SILVA ${ }^{1}$ e A. C. S. C. TEIXEIRA ${ }^{1}$ \\ ${ }^{1}$ Escola Politécnica da Universidade de São Paulo, Departamento de Engenharia Química \\ E-mail para contato: marpradosilva@usp.br
}

\begin{abstract}
RESUMO - Os processos fotoquímicos oxidativos avançados têm sido considerados para o tratamento de água e efluentes contendo o herbicida atrazina (ATZ). Neste estudo, investigamos o papel das espécies reativas de oxigênio (ROS) na fotólise da ATZ. Foram realizados experimentos na presença de $\mathrm{NaN}_{3}$ (supressor de oxigênio singlete, ${ }^{1} \mathrm{O}_{2}$ ), na presença de $\mathrm{C}_{3} \mathrm{H}_{8} \mathrm{O}$ (supressor de radicais hidroxila), na ausência de oxigênio dissolvido e em solução saturada de $\mathrm{O}_{2}$. Os resultados sugerem que a transferência de energia ou elétrons das moléculas excitadas de ATZ para as moléculas oxigênio em solução parece ser eficaz para a geração de oxigênio singlete ou espécies radicalares, respectivamente, espécies reativas que permitem oxidar o poluente em solução. Além disso, os resultados indicam que as ROS desempenham um papel importante na degradação foto-induzida da ATZ e que o oxigênio em excesso extingue o estado excitado da atrazina. Estes resultados contribuem para entender o comportamento da degradação da atrazina em diferentes processos fotoquímicos impulsionados por radiação UV, nos quais a fotólise e a oxidação por ROS podem ocorrer simultaneamente. Tais sistemas reacionais podem ser aplicados para descontaminação de água gerada na agroindústria (lavagem de equipamentos, tanques e produtos agrícolas), para tratamento de água para reuso e eventualmente de água para abastecimento.
\end{abstract}

\section{INTRODUÇÃO}

Os pesticidas são poluentes prioritários de especial interesse devido ao seu fácil transporte no meio ambiente, bem como o seu potencial de ameaçar seriamente o ambiente aquático e a saúde humana, muitos sendo considerados desreguladores endócrinos (Hincapié et al., 2005).

Dentre os pesticidas mais utilizados no mundo está a classe dos herbicidas triazínicos (Zhao et al., 2008), na qual econtra-se a atrazina (6-cloro-N-etil-N'-isopropil-1,3,5-triazina-2,4-diamina, Figura 1), um dos dez pesticidas mais comercializados no Brasil (IBAMA, 2009) e identificada em efluentes industriais e águas superficiais de diferentes regiões do Brasil e do mundo (Curini et al., 2001; Carabias et al., 2003; Ochi et al., 2006; Planas et al., 2006). 


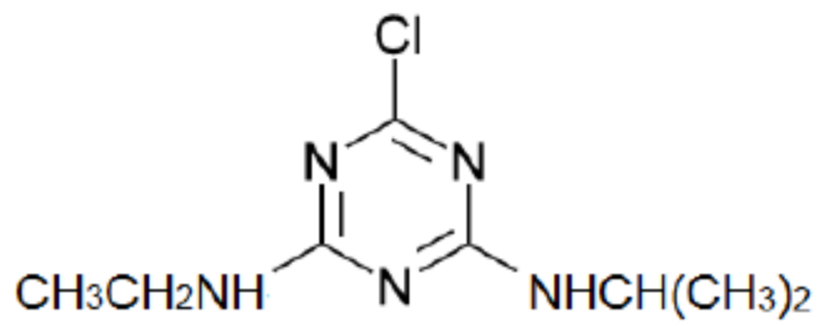

Figura 1- Fórmula estrutural da atrazina (6-cloro-N-etil-N'-isopropil-1,3,5-triazina-2,4diamina).

O pesticida atrazina se tornou um poluente de grande interesse ambiental por possuir baixa biodegradabilidade e alto potencial de contaminação de águas superficiais e subterrâneas. Dessa forma, diversos trabalhos recentes têm se dedicado ao estudo da degradação deste herbicida por meio de POA como $\mathrm{TiO}_{2} / \mathrm{UV}$ (Pérez, 2006), Fenton (Barreiro,2005), foto-Fenton e ozônio (Farré et al., 2007). Entretanto, não são encontrados na literatura estudos detalhados da fotólise da atrazina, considerando as espécies reativas que participam da degradação.

As reações fotoquímicas desempenham um papel importante no processo de degradação dos poluentes. A degradação fotolítica de contaminantes orgânicos ocorre através de dois mecanismos principais: fotólise direta e indireta. A fotólise direta refere-se à excitação de moléculas do poluente mediante a absorção de fótons, podendo resultar em reações fotoquímicas. A fotólise indireta referese à formação de intermediários reativos, que podem sensibilizar a degradação do poluente. Estes intermediários reativos, também chamados espécies reativas de oxigênio (ROS), incluem, entre outras espécies, o radical hidroxila $\left(\mathrm{HO}^{\circ}\right)$, oxigênio singlete $\left(\mathrm{O}_{2}\right)$, os radicais peroxil e peróxido de hidrogênio $\left(\mathrm{H}_{2} \mathrm{O}_{2}\right)$ (Lee, 2013).

Neste estudo foi investigado o papel das espécies reativas de oxigênio (ROS) na fotólise da atrazina, sob radiação UV a $254 \mathrm{~nm}$.

\section{MATERIAIS E MÉTODOS}

\subsection{Reagentes}

As soluções foram preparadas usando água purificada por osmose inversa (Mili-Q). Atrazina grau técnico (ATZ, MM = 215,68 $\mathrm{g} \mathrm{mol}^{-1}$, pureza mínima 92\%) foi fornecido pela Syngenta Brasil. Azida de sódio e isopropanol foram também empregados, tal como descrito mais tarde. Para a análise de HPLC, acetonitrila (qualidade HPLC) e ácido acético glacial (100\%) foram adquiridos da Merck.

\subsection{Procedimentos}

Para investigar a presença de oxigênio singlete e de radicais reativos foram realizados 
experimentos em um reator fotoquímico tubular com escoamento anular (Figura 2), que consiste em um tubo externo em vidro borossilicato equipado com uma lâmpada de vapor de mercúrio tubular de baixa pressão e concêntrica, com emissão em $254 \mathrm{~nm}$ e potência radiante de $75 \mathrm{~W}$. A temperatura do reator foi mantida em $25^{\circ} \mathrm{C}$ utilizando-se um banho termostático. $\mathrm{O}$ campo de radiação no interior do reator fotoquímico é considerado uniforme ao longo da direção axial, mas não ao longo da coordenada radial.

Os experimentos foram realizados em solução de $5 \mathrm{mg} \mathrm{L}^{-1}$ de atrazina na presença de azida de sódio $\left(\mathrm{NaN}_{3}\right.$, supressor de oxigênio singlete) ou 2-propanol $\left(\mathrm{C}_{3} \mathrm{H}_{8} \mathrm{O}\right.$, supressor de radicais hidroxila), em concentrações de 7,7 mg L $\mathrm{mg}^{-1} 14 \mathrm{mg} \mathrm{L}^{-1}$, respectivamente. Estas concentrações foram escolhidas de modo que não ocorresse competição por fótons entre a azida e a ATZ e também com base em informações da literatura.

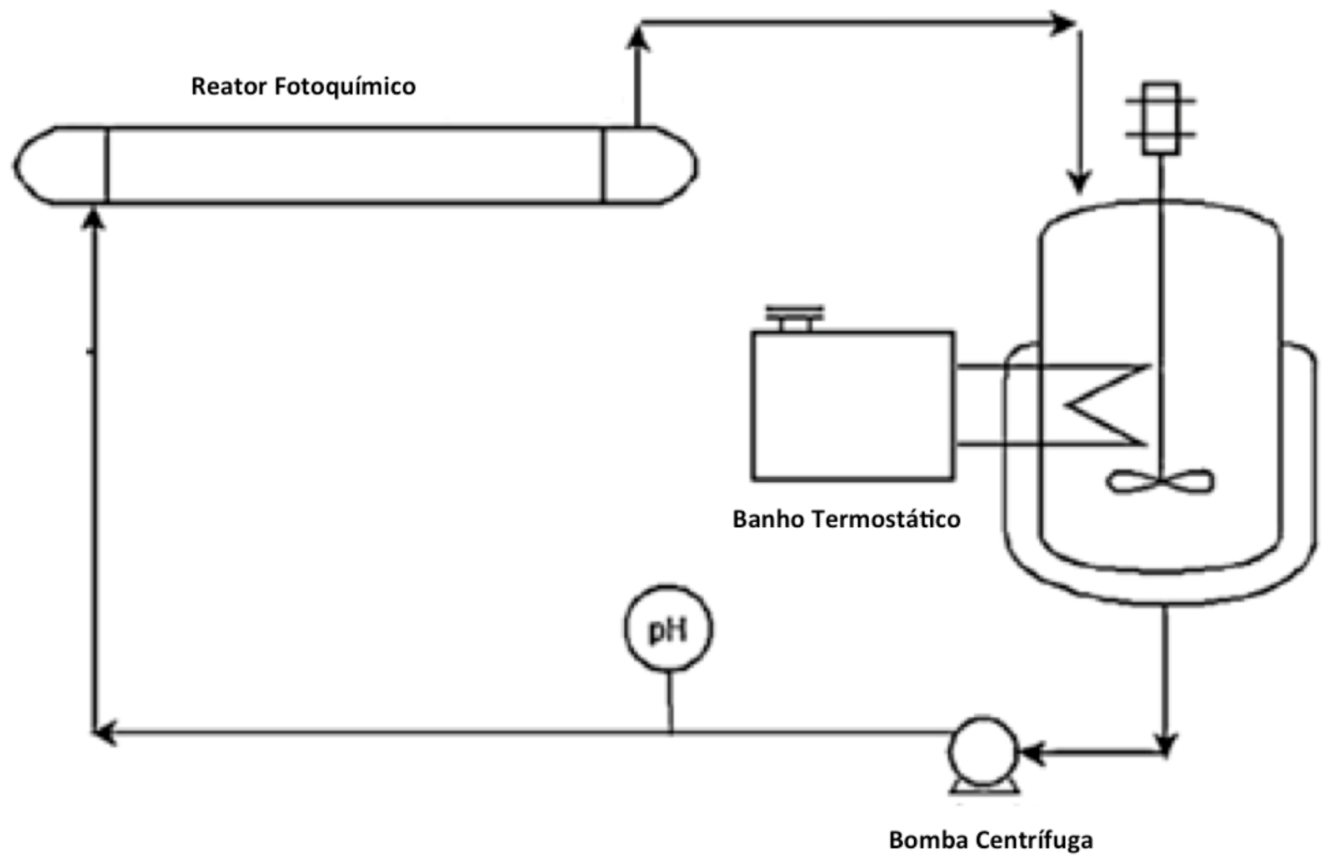

Figura 2- Esquema do reator tubular e equipamentos acessórios utilizados.

Para investigar o efeito do oxigênio dissolvido na degradação ATZ, experimentos de fotólise foram realizados em frascos de $250 \mathrm{~mL}$, utilizando uma lâmpada de $11 \mathrm{~W}$ na ausência de oxigênio dissolvido, fazendo borbulhar nitrogênio $(99,5 \%)$ durante 60 minutos antes e durante a irradiação; outro experimento foi realizado em solução saturada de $\mathrm{O}_{2}$ borbulhando-se oxigênio $(99,5 \%)$ na solução. Todos os experimentos foram realizados em triplicata. 


\section{RESULTADOS}

Para que ocorra a fotólise direta de um contaminante, este deve absorver a radiação incidente em um comprimento de onda adequado. As etapas mais comuns em reações fotoquímicas iniciadas por estados excitados são resumidos pelas equações (1) a (6) (Parsons, 2005).

$$
\begin{aligned}
& \mathrm{AB}+h v \rightarrow \mathrm{AB}^{*} \\
& \mathrm{AB}^{*} \rightarrow\left(\mathrm{A}^{\cdot} . . . \mathrm{B}\right)_{\text {cage }} \rightarrow \mathrm{A}^{\cdot}+\cdot \mathrm{B} \\
& (\mathrm{A} \cdot . . \mathrm{B})_{\text {cage }} \rightarrow \mathrm{AB} \\
& \mathrm{AB}^{*} \rightarrow\left(\mathrm{A}^{+} \ldots\right)_{\text {cage }} \rightarrow \mathrm{A}^{+}+\mathrm{B}^{-} \\
& \mathrm{AB}^{*}+{ }^{3} \mathrm{O}_{2} \rightarrow \mathrm{AB}^{* \cdot}+\mathrm{O}_{2}^{-} \\
& \mathrm{AB}^{*}+{ }^{3} \mathrm{O}_{2} \rightarrow \mathrm{AB}+{ }^{1} \mathrm{O}_{2}
\end{aligned}
$$

$\mathrm{O}$ estado eletronicamente excitado $\mathrm{AB}^{*}$ gerado a partir de absorção de luz é altamente energético. A clivagem homolítica da ligação química (2) corresponde ao passo de reação predominante. Quando os radicais escapam do "envoltório" de moléculas de solvente (solvent cage), podem participar de reações com espécies em solução. Por outro lado, a recombinação dos radicais primários (3) tem alta probabilidade de ocorrer, o que explica o baixo rendimento quântico observado em alguns casos. Uma alternativa à cisão homolítica é a cisão heterolítica no estado excitado (4), o que é favorecido em solventes polares, tais como água (Nick et al., 1992). A transferência de elétrons e de energia para o $\mathrm{O}_{2}$ é também possível, mas requer um estado excitado de longa duração como o estado triplete. Espécies reativas de oxigênio (ROS), tais como o radical superóxido (5) e oxigênio singlete, ${ }^{1} \mathrm{O}_{2}$ (6) são gerados (Parsons, 2005).

Para investigar qualitativamente o papel das ROS, soluções contendo ATZ foram irradiados na presença de azida de sódio, um supressor seletivo para ${ }^{1} \mathrm{O}_{2}$, de acordo com Razavi et al. (2011) e outros autores, ou 2-propanol, um conhecido supressor de radicais hidroxila (Xu et al., 2011). Os resultados são mostrados na Figura 3. 


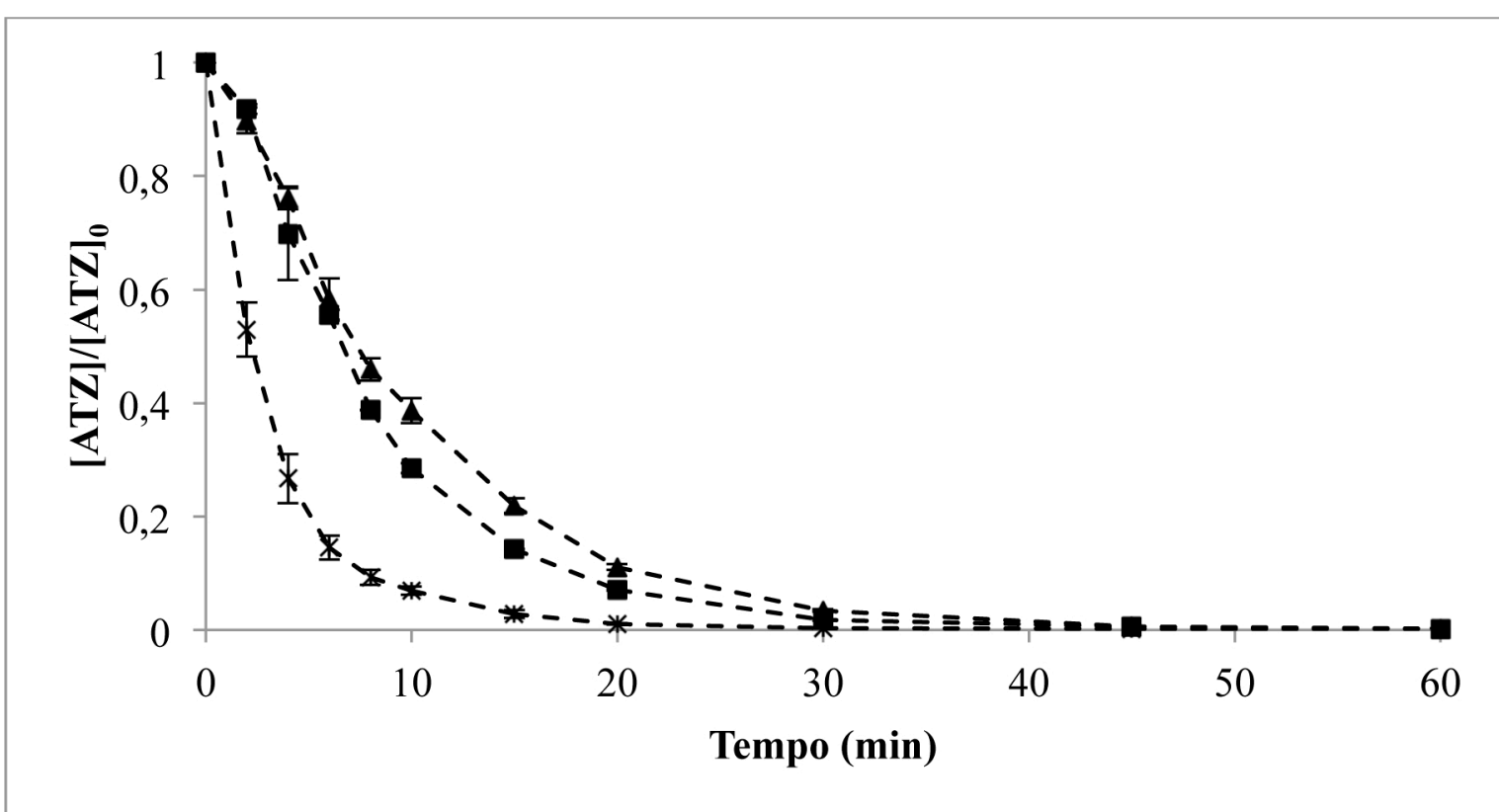

Figura 3 - Degradação da atrazina (ATZ) durante o processo de fotólise em $\mathrm{pH} 6$, [ATZ $]_{0}=5 \mathrm{mg}$ $\mathrm{L}^{-1}$ e $E_{\mathrm{P}, 0}=3.6 \times 10^{18}$ fótons $\mathrm{L}^{-1} \mathrm{~s}^{-1}$. $* \mathrm{UV} ; \leftarrow \mathrm{UV}+2$-propanol; $\rightarrow-\mathrm{UV}+\mathrm{NaN}_{3}$.

A adição do ânion azida resultou em diminuição na taxa de degradação ATZ $\left(\mathrm{k}=1,09 \times 10^{-1}\right.$ $\min ^{-1}, \mathrm{t}_{1 / 2}=7 \mathrm{~min}$ ), sugerindo assim que a transferência de energia das moléculas excitadas de ATZ para as moléculas de ${ }^{3} \mathrm{O}_{2}$ parece ser eficaz para gerar espécies de ${ }^{1} \mathrm{O}_{2}$, de acordo com a reação (6). Em um estudo anterior, Oliva et al. (2005) obtiveram o espectro de fosforescência ATZ e o tempo de vida de seu estado excitado na água. A existência de tal estado é um pré-requisito para as reações de foto sensibilização. Assim, embora se espere que o estado excitado seja de curta duração em temperatura ambiente, a degradação da ATZ promovida pelo ${ }^{1} \mathrm{O}_{2}$ não pode ser descartada. Como mostrado na Figura 3, a adição de 2-propanol também resultou num decréscimo da velocidade de degradação ATZ $\left(\mathrm{k}=1,1 \times 10^{-1} \mathrm{~min}^{-1}, \mathrm{t}_{1 / 2}=8 \mathrm{~min}\right)$, sugerindo que o estado excitado da molécula de ATZ pode transferir elétrons para o oxigênio dissolvido e induzir a formação de espécies que podem oxidar o poluente alvo (5).

Os resultados na Figura 4 mostram que em meio livre de oxigênio a taxa de degradação foi menor $\left(\mathrm{k}=5 \times 10^{-2} \mathrm{~min}^{-1}, \mathrm{t}_{1 / 2}=15 \mathrm{~min}\right)$, devido à impossibilidade de formação de ROS, indicando que estas espécies têm um papel importante na degradação foto-induzida da ATZ. $\mathrm{O}$ fato da fotodegradação/UV da ATZ não ter sido completamente inibida por remoção de oxigênio indica a importância também da fotólise direta na degradação ATZ a $254 \mathrm{~nm}$. Por outro lado, quando o meio reacional foi saturado com oxigênio, a degradação da ATZ também diminuiu $\left(\mathrm{k}=4,1 \times 10^{-2} \mathrm{~min}^{-1}\right.$, $\mathrm{t}_{1 / 2}=19 \mathrm{~min}$ ). Uma possível explicação é que o oxigênio em excesso iniba o estado excitado da atrazina, de tal forma que as reações (5) e (6) substituam as reações (2) e (4). Resultados similares foram obtidos por Nick (1992). 


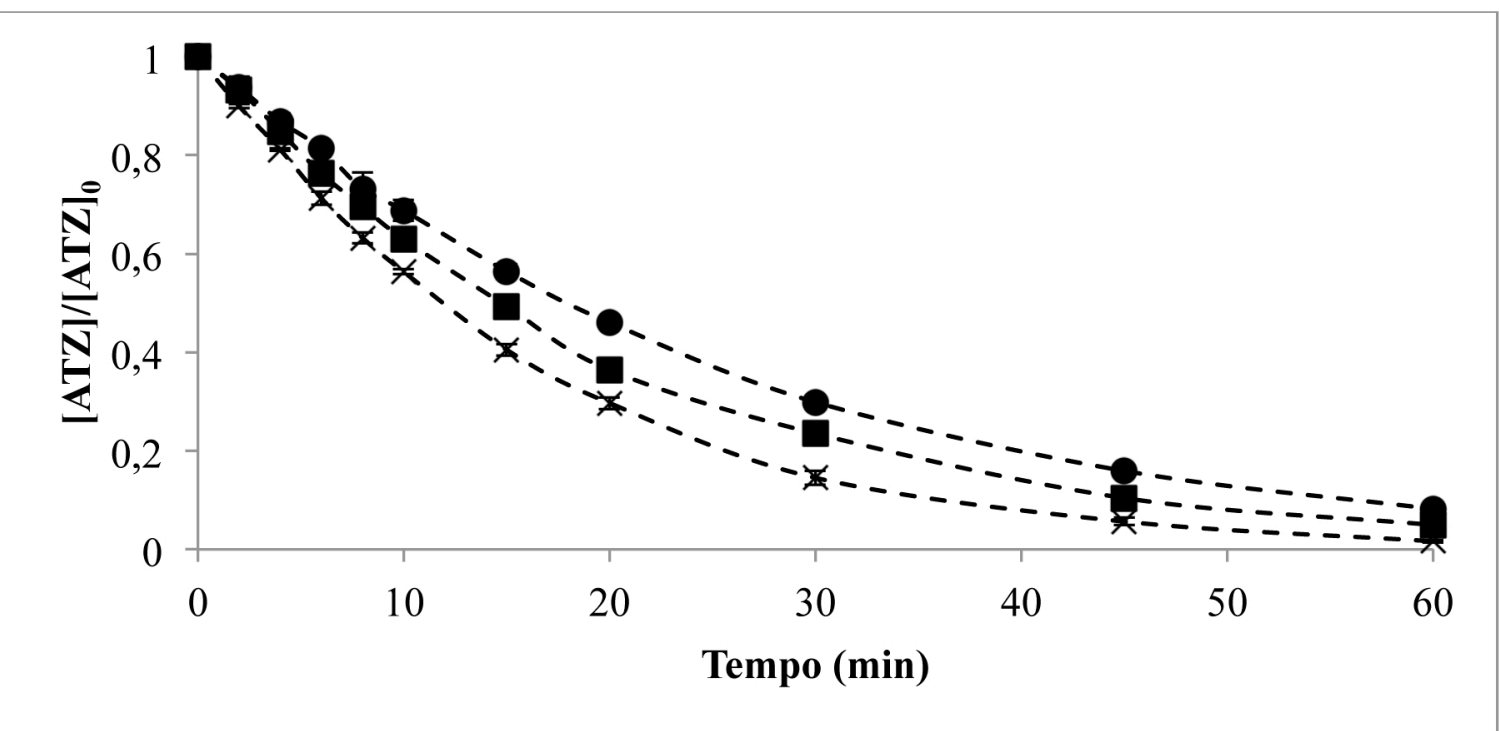

Figura 4 - Degradação da atrazina (ATZ) durante o processo de fotólise em $\mathrm{pH} 6,[\mathrm{ATZ}]_{0}=5 \mathrm{mg}$ $\mathrm{L}^{-1}$. * UV; - - UV + solução borbulhada com nitrogênio; $\rightarrow$ - UV + solução saturada com oxigênio.

\section{CONCLUSÕES}

Neste estudo, estudou-se mais detalhadamente a fotólise da atrazina (ATZ) em solução aquosa sob radiação UV de $254 \mathrm{~nm}$. Além da fotólise direta de moléculas do pesticida a partir de absorção de fótons nesse comprimento de onda, foi possível verificar que as espécies reativas de oxigênio (ROS) têm papel importante na degradação foto-induzida da ATZ e que o oxigênio em excesso inibe o estado excitado deste pesticida. Estes resultados são úteis em estudos de degradação de atrazina em processo de oxidação foto-induzida, nos quais a fotólise e a oxidação por ROS podem ocorrer simultaneamente. Guardadas questões de escala, que devem ser avaliadas adequadamente, tais sistemas reacionais são potencialmente úteis para descontaminação de água gerada na agroindústria (lavagem de equipamentos, tanques e produtos agrícolas), para tratamento de água para reuso e possivelmente de água para abastecimento.

\section{REFERÊNCIAS}

BARREIRO, J. C. Estudo do mecanismo de oxidação do herbicida Atrazina na presença de minerais e substâncias húmicas em ambiente anaeróbio, Tese de D.Sc., Universidade Federal de São Carlos, São Carlos, 2005.

CARABIAS-MARTIINEZ, R.; RODRÍGUEZ-GONZALO E.; REVILLA-RUIZ P. Determination of endocrine-disrupting compounds in cereals by pressurized liquid extraction and liquid chromatography-mass spectrometry Study of background contamination. Journal of Chromatography $A$, v.1137, p.207-215, 2006. 
CURINI, R.; GENTILI, A.; MARCHESE, S.; et al. Solid phase extraction followed by highperformance liquid chromatography-ionspray interface-mass spectrometry for monitoring of herbicides in environmental water. Journal Chromatography A, v. 874, n. 2, p. 187-198, 2000.

FARRÉ, M. J.; FRANCH, M. I.; AYLLÓN, et al. Biodegradability of treated aqueous solutions of biorecalcitrant pesticides by means of photocatalytic ozonation, Desalination, v. 211, p. 22-33, 2007.

HINCAPIÉ, M.; MALDONADO, M.; OLLER, I. GERNJAK, W.; SANCHEZ-PEREZ, J.; BALLESTEROS, M.; MALATO, S. Solar photocatalytic degradation and detoxification of EU priority substances. Catalysis Today, v. 101, p. 203-210, 2005.

LEE, E.; Glover C. M.; Rosario-Ortiz F. O. Photochemical Formation of Hydroxyl Radical from Effluent Organic Matter: Role of Composition. Environmental Science \& Technology, v. 47, p.1207312080, 2013.

NICK K.; SCHOLER H. F.; MARK G.; SOYLEMEZ T.; AKHLAQ M. S.; SCHUCHMANN H. P.; VON SONNTAG C. Degradation of some triazine herbicides by UV radiation such as used in the UV disinfection of drinking water. Journal of Water SRT-Aqua v.41, p.82-87, 1992.

OCHI, B. C.; BRITO, V. F.; MEDEIROS , M. A. C. Avaliação da contaminação de águas superficiais por herbicidas e nutrientes e em áreas com o cultivo de cana-de-açúcar no município de iracemápolis, UNICAMP/CESET, Campinas, 2006.

OLIVA J. M.; AZENHA M. E. D. G.; BURROWS H. D.; COIMBRA R.; MELO J. S.; CANLE L. M.; FERNÁNDEZ M. I.; SANTABALLA J. A.; SERRANO-ANDRÉS L. (2005) On the low-lying excited states of sym-triazine-based herbicides. ChemPhysChem, v.6, p. 306-314, 2005.

PARSONS, S. Advanced Oxidation Processes for Water and Wastewater Treatment. IWA Publishing, London, 2005.

PÉREZ, M. H.; PEÑUELA G.; MALDONADO M. I.; et al. Degradation of pesticides in water using solar advanced oxidation processes. Applied Catalysis B: Environmental, v. 64, 272-281, 2006.

PLANAS, C.; PUIG, A.; RIVERA, J.; CAIXACH, J. Analysis of pesticides and metabolites in 
Spanish surface waters by isotope dilution gas chromatography/mass spectrometry with previous automated solid-phase extraction Estimation of the uncertainty of the analytical results. Journal of Chromatography A, v.1131, p.242-252, 2006.

RAVAZI, B.; BEN ABDELMELEK, S.; SONG, W.; O'SHEA, K. E.; COOPER, W. J.; Photochemical fate of atorvastatin (lipitor) in simulated natural waters. Water Research v.45, p. 625$631,2011$.

XU, H.; COOPER, W. J.; JUNG, J.; SONG, W. Photosensitized degradation of amoxicillin in natural organic matter isolate solutions. Water Research, v. 45, p. 632-638, 2011. 